\title{
Early Prediction of Transient Voltage Sags caused by Rotor Swings
}

Tilman Weckesser
Hjörtur Jóhannsson
Thierry Van Cutsem

\begin{abstract}
The paper investigates various methods to predict voltage sags at load buses caused by large generator rotor swings and following a transient disturbance. Three different prediction methods are proposed, which all use real-time measurements from PMUs. One of the methods uses a slightly extended version of the E-SIME method. The other two methods use measurements and process them by recursive least square estimation. It is shown that the prediction method employing E-SIME allows the earliest detection of a critical voltage sag with satisfactory accuracy.

Index Terms-power system stability, rotor swings, transient stability, voltage sag prediction
\end{abstract}

\section{INTRODUCTION}

$\mathbf{T}$ HE primary cause for voltage sags/dips is the occurrence of a fault. In the literature on power quality voltage sags/dips is a topic vastly addressed [1]. A rarely addressed reason for voltage sags is angular separation of generators or rotor angle swing resulting from a fault. Certain fault scenarios cause large rotor angle displacements, which result in transiently low voltages for which the system response should be considered unacceptable. However, from a rotor angle stability viewpoint, such a scenario may be assessed transiently stable, since the generators remain in synchronism.

Voltages falling below a critical level can cause subsequent events such as unintentional load tripping, which may further deteriorate the system condition. An early and accurate detection of an unacceptable voltage sag can be used to trigger appropriate control actions and avoid such detrimental events.

The prediction of this type of voltage sag using the Transient Energy Function was described in the early reference [2]. In [3] sensitivities relative to voltage dips were derived using this method. The sensitivities relate the voltage sag depths to certain parameters such as terminal voltages and power generation. However, approaches using the transient energy function always suffer from restrictions placed on the power system model. In [4] the authors treated the transient voltage dip acceptability problem using a two-dimensional table of critical voltage level and critical voltage dip duration. The paper also analyzed the issue of transient voltage stability of dynamic loads such as induction machines. In the recent publication [5], the authors developed a method for contingency filtering and ranking with respect to voltage dips using sensitivities. A survey of current practices for transient voltage sag criteria related to power system stability was presented in [6].

T. Weckesser and H. Jóhannsson are with the Technical University of Denmark, Kgs. Lyngby, Denmark, email: \{jtgw $\}\{$ hjjo $\}$ @elektro.dtu.dk

T. Van Cutsem is with the Fund for Scientific Research (FNRS) at the University of Liège, Belgium, email: t.vancutsem@ulg.ac.be

Research supported by the Danish Council for Strategic Research (DSF).
In the present paper voltage sags are investigated with focus on system dynamics rather than power quality. The aim is to derive a method, which in real-time allows early and accurate prediction of voltage sags caused by angular separation. To this purpose, different approaches have been implemented and their performance is assessed. In order to avoid limitations due to the model or data, the proposed methods utilize synchronized phasor measurements.

\section{Voltage SAg PREdiction}

In this section three approaches are described, which intend to early predict an imminent voltage sag. These approaches will later be compared and benchmarked against time-domain simulation. It should be noted that all the proposed methods use wide-area measurements, which introduce a delay due to the communication time needed to collect the measurements.

\section{A. Modelling for voltage sag prediction}

For voltage sag prediction, the generators are modelled using the so-called "classical" model [7]. This corresponds to a representation of each generator by an e.m.f. $\bar{E}^{\prime}$ of constant magnitude behind the transient reactance $X_{d}^{\prime}$ while loads are converted to constant shunt admittances. The reason for using this very simple model is threefold. First, the time frame of concern is short, in the order of one second after fault clearing. Second, the model is only used to get a fast estimation of the voltage sag. Third, the method utilizes realtime measurements from phasor measurement units (PMUs) to fit the parameters of the classical model to the current operating point. To simplify matters the Norton equivalent, i.e. a current source $\bar{E}^{\prime} /\left(j X_{d}^{\prime}\right)$ in parallel with the admittance $1 /\left(j X_{d}^{\prime}\right)$, is chosen to represent each generator. Under these assumptions, the following well-known algebraic equations can be used:

$$
\overline{\boldsymbol{I}}=\boldsymbol{Y} \overline{\boldsymbol{V}}
$$

where the vector of complex current injections is $\bar{I}$, the vector of complex bus voltages is $\overline{\boldsymbol{V}}$, and $\boldsymbol{Y}$ is the "augmented" bus admittance matrix, which is obtained by adding the transient reactances of the generators and shunt admittances of the loads to the admittance matrix of the network.

The number of buses is $n$ and the number of generators is $m$. The bus entries are sorted so that the buses where generators are connected have the indices from $n-m+1$ to $n$. Hence, 
Eq. (1) can be written as:

$$
\left[\begin{array}{c}
0 \\
\vdots \\
0 \\
\bar{E}_{1}^{\prime} /\left(j X_{d, 1}^{\prime}\right) \\
\vdots \\
\bar{E}_{m}^{\prime} /\left(j X_{d, m}^{\prime}\right)
\end{array}\right]=\boldsymbol{Y}\left[\begin{array}{c}
\bar{V}_{1} \\
\vdots \\
\bar{V}_{n-m} \\
\bar{V}_{n-m+1} \\
\vdots \\
\bar{V}_{n}
\end{array}\right]
$$

where the zero sub-vector has dimension $n-m$ and $\bar{V}_{i}$ is the complex voltage at the $i$-th bus.

\section{B. Voltage Sag Prediction using PMU Voltage Measurements}

The first method to predict the depth of a voltage sag consists in acquiring bus voltage measurements in the postfault configuration and computing a quadratic approximation of each voltage evolution. To this purpose a Recursive Least Square (RLS) estimator with exponential forgetting factor [8] is used together with the linear regression model.

$$
\mathbf{V}_{i}=\Theta_{i}^{T} \mathbf{x}_{i}
$$

where $\mathbf{x}_{i}$ is the regressor $\left[t_{i}^{2}, t_{i}, 1\right]^{T}$ at a discrete time, $\mathbf{V}_{i}$ is the observation, which is a vector of the measured bus voltages at time $t_{i}$, and $\boldsymbol{\Theta}_{i}$ is the corresponding parameter matrix determined at $t_{i}$ :

$$
\boldsymbol{\Theta}_{i}=\left(\begin{array}{ccc}
a_{1} & \ldots & a_{l} \\
b_{1} & \ldots & b_{l} \\
c_{1} & \ldots & c_{l}
\end{array}\right)
$$

The first three sets of voltage magnitudes are used to compute a first estimate of the parameter matrix, which holds all the parameters of the quadratic approximations of all $l$ load bus voltages. This is achieved through linear least square approximation. Additionally, the covariance matrix is computed, which is used in the RLS estimator and needs to be updated at each step. The covariance matrix $\mathbf{R}$ is initialized as:

$$
\mathbf{R}_{i}=\mathbf{X}^{T} \mathbf{X}
$$

where $\mathbf{X}$ is the regression matrix holding the regression vectors $\mathbf{x}_{k}^{T}, k=i-2, i-1, i$. The parameter matrix $\boldsymbol{\Theta}$ is updated by the RLS estimator as follows:

$$
\boldsymbol{\Theta}_{i}=\boldsymbol{\Theta}_{i-1}+\mathbf{R}_{i}^{-1} \mathbf{x}_{i}\left(\mathbf{V}_{i}-\mathbf{x}_{i}^{T} \boldsymbol{\Theta}_{i-1}\right)
$$

The inverse of the covariance matrix is updated as follows:

$$
\mathbf{R}_{i}^{-1}=\lambda^{-1}\left[\mathbf{R}_{i-1}^{-1}-\mathbf{R}_{i-1}^{-1} \mathbf{x}_{i}\left(\lambda+\mathbf{x}_{i}^{T} \mathbf{R}_{i-1}^{-1} \mathbf{x}_{i}\right)^{-1} \mathbf{x}_{i}^{T} \mathbf{R}_{i-1}^{-1}\right]
$$

Consequently, only one time at the beginning the inverse of the covariance matrix has to be determined and, thereafter, it solely is updated using (7), which does not involve inverting a matrix.

In [9] a typical range for the forgetting factor is stated as $\lambda=0.95 \ldots 0.995 . \lambda$ equal to one implies that all measurements are "remembered" and considered in the computation of the parameters. Due to the strong non-linear equations describing the dynamics of the power system, it was chosen to use a factor at the lower limit of the common range $(\lambda=0.95)$.
The RLS estimator determines from the voltage magnitude measurement for each load bus $k$ a function $V_{k}(t)$.

$$
V_{k}(t)=a_{k} t^{2}+b_{k} t+c_{k}
$$

This approximated function of the voltage is then used to predict the depth of the voltage sag through an assessment of its extreme values.

\section{Voltage Sag Prediction using the phase angle of $E^{\prime}$}

Since the voltage sag in this study originates from a deviation of the rotor angles of a group of generators, the second proposed method uses an RLS estimator to predict the individual rotor angle evolution. Thus, the rotor angle of the $k$-th generator is obtained as:

$$
\delta_{k}(t)=a_{k} t^{2}+b_{k} t+c_{k}
$$

In order to do so, the method computes from each acquired set of synchronized bus voltage and current measurements the matching operating point for each generator, when represented by the classical model. The phase angle $\delta$ of the computed e.m.f. $\bar{E}^{\prime}$ corresponds to the rotor angle of the machine and its evolution may be predicted with an RLS estimator, as introduced in the previous section. For the period, where the rotor angle evolution is sufficiently described by the quadratic approximation of Eq. (9) and under the assumption of constant magnitude of the e.m.f.'s, the load bus voltages can be computed by solving:

$$
\left[\begin{array}{c}
0 \\
\vdots \\
0 \\
\bar{E}_{1}^{\prime} e^{j \Delta \delta_{1} /\left(j X_{d, 1}^{\prime}\right)} \\
\vdots \\
\bar{E}_{m}^{\prime} e^{j \Delta \delta_{m} /\left(j X_{d, m}^{\prime}\right)}
\end{array}\right]=\boldsymbol{Y}\left[\begin{array}{c}
\bar{V}_{1}^{\dagger} \\
\vdots \\
\bar{V}_{n-m}^{\dagger} \\
\bar{V}_{n-m+1}^{\dagger} \\
\vdots \\
\bar{V}_{n}^{\dagger}
\end{array}\right]
$$

where $\Delta \delta$ corresponds to changes in rotor angle from the current state to a state some time ahead. In this work the rotor angle prediction was performed over the next $300 \mathrm{~ms}$.

\section{Voltage Sag Prediction using E-SIME}

1) E-SIME Method: The SIngle Machine Equivalent (SIME) Method is a transient stability assessment method based on the Equal-Area Criterion (EAC). A detailed description of the method can be found in [10]. A recent discussion of the achievements and prospects of Emergency-SIME (ESIME) is available in [11]. The methodology of SIME relies on the possibility of representing the post-fault dynamics of a multi-machine power system by a suitable One-Machine Infinite Bus (OMIB) equivalent. The transient stability of the OMIB can then be assessed applying EAC.

In E-SIME real-time measurements are collected in the post-fault configuration at regular time steps $t_{i}$. These measurements are used to formulate an OMIB equivalent, which represents the dynamics between a group of "Critical" Machines (CMs) and a group of "Non-critical" Machines (NMs). The OMIB is characterized by its rotor angle $\delta$, speed $\omega$, 
inertia coefficient $M$ and acceleration power $P_{a}$, which is the difference between mechanical and electrical power. These parameters and variables can be computed from machine parameters and appropriate aggregation of the CMs and NMs.

Subsequently, the EAC allows to compute a transient stability margin $\eta$, which is negative for an unstable and positive for a stable case [10]:

$$
\eta=-\int_{\delta_{i}}^{\delta_{u}} P_{a} d \delta-1 / 2 M \omega_{i}^{2}
$$

where $\delta_{i}$ and $\omega_{i}$ correspond to $\delta\left(t_{i}\right)$ and $\omega\left(t_{i}\right)$ and $\delta_{u}$ is the angle where the following instability conditions are met:

$$
P_{a}\left(\delta_{u}\right)=0 \text { and } \dot{P}_{a}\left(\delta_{u}\right)>0
$$

In E-SIME the angle $\delta_{u}$ is estimated through a prediction of the $P_{a}(\delta)$-curve of the OMIB. To this purpose, three successive data sets of the OMIB, consisting of $P_{a}$ and $\delta$, are used to compute a quadratic approximate of the curve:

$$
P_{a}(\delta)=a \delta^{2}+b \delta+c
$$

of which the parameters $a, b$ and $c$ are computed from the three data sets and, subsequently, are refined using a weighted least square technique.

The implementation of E-SIME comprises the following steps. Step 1: Collect the first three post-fault measurement sets of the multi-machine system. Step 2: Using Taylor series expansion, predict the rotor angles of the individual machines some time ahead (e.g. $100 \mathrm{~ms}$ ). Step 3: Identify the CMs by ranking the machines according to the predicted rotor angles and searching for the maximum angular gap between two successive machines. The machines above the gap form the candidate CMs and the ones below the candidate NMs. The two groups are aggregated into two equivalent machines and, thereafter, the "candidate" OMIB can be determined. Step 4: The parameters of the OMIB from (at least) three successive data sets are utilized to estimate its $P_{a}(\delta)$-curve with Eq. (13). Step 5: Then the angle $\delta_{u}$ can be computed, if Eq. (13) meets the conditions (12). If not, a new set of data is acquired and the procedure is repeated from Step 2. If the conditions are met, the stability margin is computed utilizing Eq. (11). Then a new set of data is acquired and Steps 2 to 5 are repeated to refine the computed $\delta_{u}$ and $\eta$. The procedure is terminated when the margin converged to a constant value or the return angle is reached, where:

$$
P_{a}\left(\delta_{r}\right)<0 \text { and } \omega_{r}=0
$$

2) Prediction using E-SIME: In the following, a method is derived, which uses E-SIME to predict and early detect a voltage sag. The idea is that E-SIME uses real-time data to predict the rotor angle evolution in each time step of the postfault configuration. If the system is found stable, the method predicts the return angle of the critical cluster. This angle together with the up-to-date bus voltage measurements are then used to predict the expected minimum load bus voltage.

Just after fault clearance, the admittance matrix as described in Section II-A is computed and the assessment with E-SIME is executed. If the computed stability margin is positive, the return angle $\delta_{r}$ is computed by solving:

$$
0=\int_{\delta_{i}}^{\delta_{r}} P_{a}(\delta) d \delta-1 / 2 M \omega_{i}^{2}
$$

where $P_{a}$ is approximated by the quadratic function in Eq. (13). From the current rotor angle $\delta_{i}$ of the candidate OMIB and the predicted angle $\delta_{r}$, the maximum angular deviation of the CMs relative to the NMs can be estimated by

$$
\Delta \delta_{r}=\delta_{r}-\delta_{i}
$$

Since it is assumed that the voltage sag origins from the rotor swing of the generators, it is expected that the voltage sag minimum will be reached when the group of CMs and the group of NMs have reached there maximum angular separation [2], which occurs at the return angle. The operating point of the synchronous machine represented by the classical model is computed by utilizing the most recent bus voltage PMU measurements and solving Eq. (2). Hence, the minimum bus voltages are computed by assuming constant magnitude of the e.m.f.'s, but a rotation of $\bar{E}^{\prime}$ of the CMs by $\Delta \delta_{r}$. Then the bus voltages at $\delta_{r}$ can be computed by solving Eq. (2).

\section{Results For Voltage Sag PRediction}

\section{A. Test system and test case}

1) Test system: In order to validate the proposed prediction methods and to assess their accuracy, the well-known New England \& New York system [12] is used. The system is composed of 68 buses and 16 generators. In the time-domain simulations the 33 loads are modelled as constant impedances. The generators are all equipped with a simple excitation and voltage regulation system, as well as a thermal turbine/governor model. Additionally, all generators, but GEN-7 and GEN-14, are equipped with power system stabilizers. The machines are modelled with four rotor windings.

2) Test case: In the test scenario a three-phase short-circuit on the transmission line connecting buses 16 and 21 occurs at time $t=1 \mathrm{~s}$. This fault is very close to bus 21 and is cleared after $150 \mathrm{~ms}$ through the opening of the breakers at both ends of the faulted transmission line. This scenario was also proposed in [12].

Due to the fault some generators accelerate relative to the others. This leads to a large angular separation of the rotors. The response of a selection of generators is shown in Fig. 1. The selection consists of the five generators with the largest increase of rotor angle (GEN-1, GEN-4, GEN-5, GEN-6 and GEN-7), the three generators with the largest decrease (GEN-14, GEN-15 and GEN-16) and one which is barely affected (GEN-13). The rotor angle evolution shows that certain generators are affected to a higher degree than others; for instance GEN-6 and GEN-7. Yet, synchronism is maintained, a new stable equilibrium is reached and the scenario can be assessed to be transiently stable. The voltage magnitudes at a selection of load buses over time are presented in Fig. 2. This selection includes the five buses which experience the deepest voltage sag (buses 15, 16, 21, 23 and 24), 


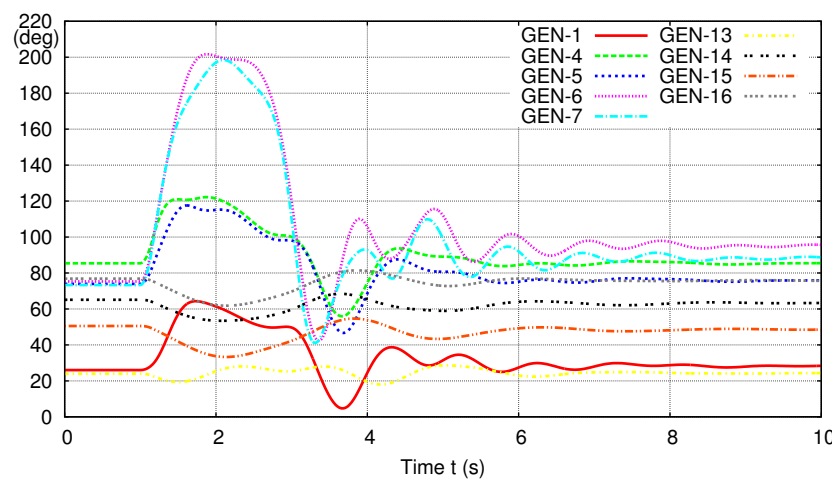

Fig. 1. Evolution of generator rotor angles (with respect to center of inertia)

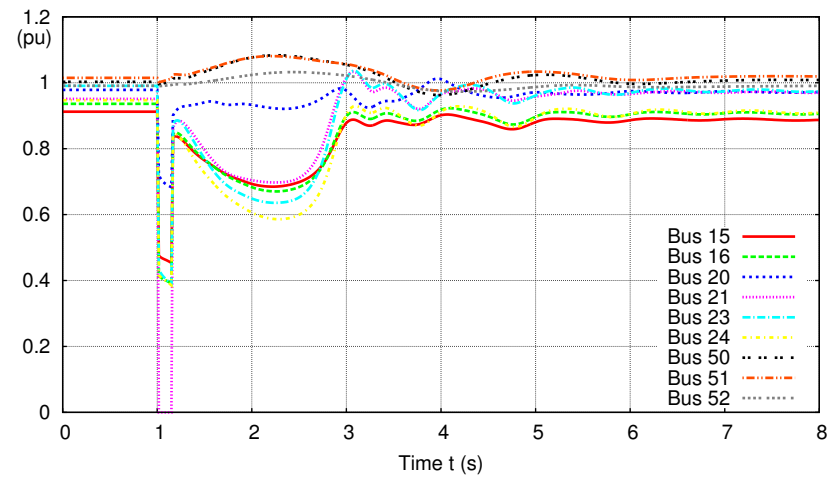

Fig. 2. Evolution of voltage magnitudes at load buses

the three buses whose voltage magnitude are slightly increased (buses 50, 51 and 52), and one bus barely affected by the post-fault dynamics (bus 20). In the fault-on period voltage magnitudes in the vicinity of the fault location experience a dramatic drop and immediately recover after fault clearance. However, in the subsequent evolution a voltage sag can be observed at certain buses with voltage dropping below the critical value of $0.7 \mathrm{pu}$, which is unacceptably low and long lasting. An early and accurate detection of these voltage sags is very valuable for a secure operation of the power systems.

\section{B. Detection of critical load buses}

In this section the different prediction methods are evaluated with respect to their ability to early and consistently predict the crossing of a critical voltage level (here taken as $0.7 \mathrm{pu}$ ). Figure 3 shows on the abscissa the simulated time and on the ordinate the loads. A data point at a certain time and for a certain load indicates that the particular method predicted, at that time instant, that the voltage at this load bus will drop below the critical level during the voltage swing.

The black crosses depict the results when using directly PMU voltage measurements and an RLS estimator (see Section II-B), the blue circles show the results using the phase angle of the e.m.f.'s and an RLS estimator (see Section II-C) and the green $\times$ 's relate to the approach using SIME (see Section II-D). Consequently, the graph allows to assess how early a critically low voltage at a certain load can be predicted and how consistent this prediction is. Furthermore, the graph

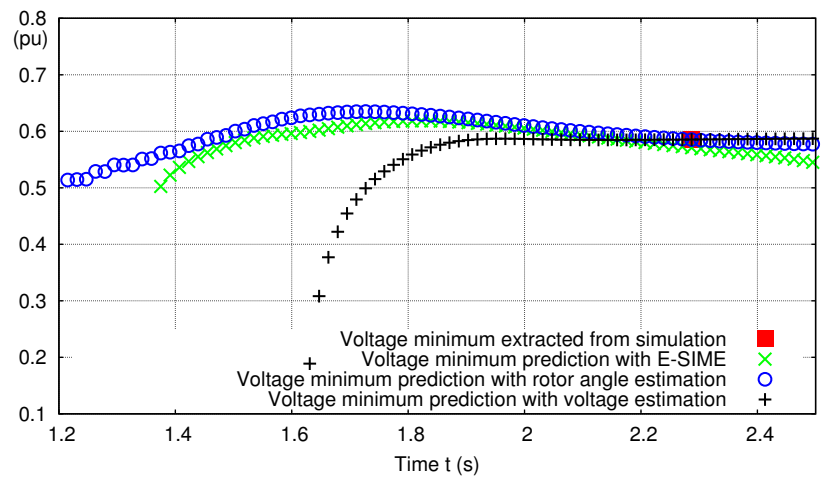

Fig. 4. Prediction of voltage minimum at bus 24 (load 14)

discloses if a method predicts critically low voltages only at those buses, where the voltage actually drops below the critical value. Finally, the red squares indicate the time instants at which the voltage of a particular load drops below the critical value in the time-domain simulation. This is the case, in the analysed scenario, for loads $8,9,12,13$ and 14 , which correspond to buses $15,16,21,23$ and 24 .

Figure 3 indicates that the method utilizing real-time voltage measurements (black crosses) needs to acquire a large number of measurements to correctly predict the critically low voltage sags, which leads to some detections taking place after the critical level has been crossed (e.g. loads 13 and 14). Furthermore, at some point the method unduly predicts low voltage for some non-critical loads (e.g. loads $2-5$ ). The second method, which uses the phase angle of the e.m.f.'s for the prediction (blue circles), is more consistent in the prediction of the critical loads. It allows an early identification, while only for a short initial period some loads are unduly flagged as critical (e.g. loads $3-5$ ). However, for some loads, the identification of critically low voltages is interrupted before the respective voltage falls below the critical level (e.g. loads 8 , 9 and 12). The results depicted by the green $\times$ 's and utilizing E-SIME are very satisfactory. Generally, only the critical loads are flagged and the identification is well before the voltage at the particular bus falls below the critical level.

\section{Earliness and Accuracy of the Prediction}

In this section, the methods are assessed with respect to their ability to early and accurately detect the actual minimum of the voltage sag observed at a particular bus. The results are presented for the bus experiencing the lowest voltage sag (bus 24 , Fig. 4) and the bus where the voltage marginally drops below the critical level (bus 21, Fig. 5). In both graphs the red squares indicate the time and value of the voltage minimum at the particular bus provided by time-domain simulation.

The prediction method which uses the bus voltages directly (black crosses) detects late that both voltages will fall below the critical value. Furthermore, the first predicted values are very pessimistic, although the predicted voltage minimum, subsequently, converges to the accurate value in both cases. The second method, which predicts the voltage sag from the phase angle of the e.m.f.'s (blue circles), early indicates a 


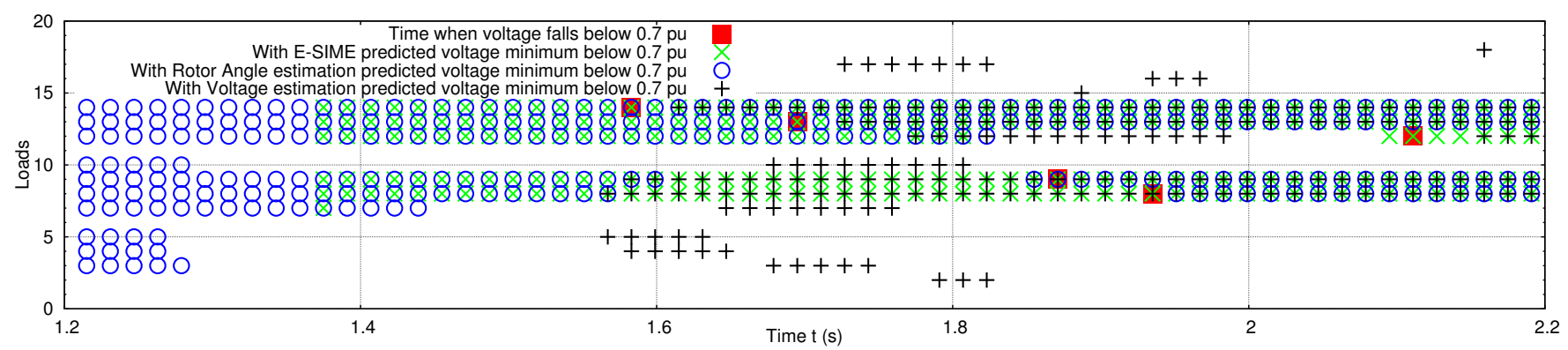

Fig. 3. Early detection of load bus voltages falling below the critical value of $0.7 \mathrm{pu}$

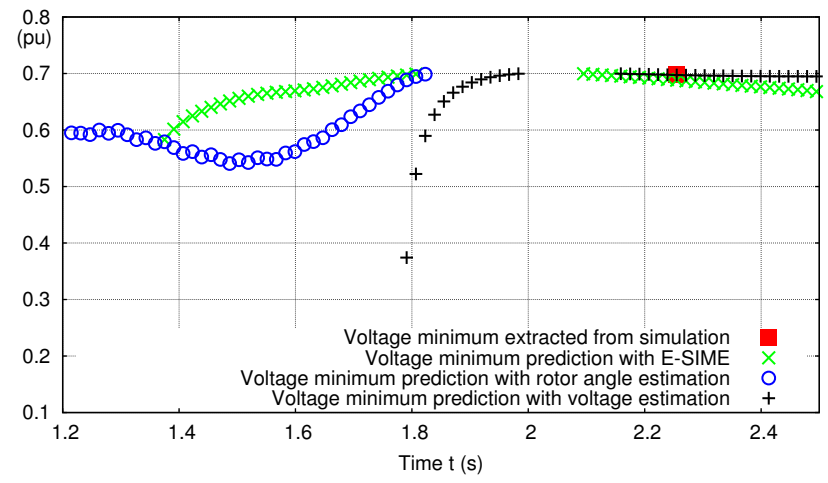

Fig. 5. Prediction of voltage minimum at bus $21(\operatorname{load} 12)$

voltage below the critical value at both buses. The maximum deviation to the actual minimum is smaller compared to the first method. However, the prediction for bus 24 is not as accurate as with the first method, when getting close to the time where the actual voltage minimum occurs. Moreover, at bus 21 the prediction is interrupted, because the voltage is no longer identified to be critical. This occurs before the actual voltage has reached its minimum and, thereafter, the method fails to again detect the shortfall of the critical voltage magnitude. The third method, which uses E-SIME for the prediction of the depth of the voltage dip (green $\times$ 's), detects the crossing of the critical voltage around $200 \mathrm{~ms}$ later than the second method, but still significantly earlier than the first method. The prediction accuracy is comparable to the second approach, but not as good as the first method. In the prediction of the minimum voltage in the marginally critical case at bus 21 , the prediction is interrupted at some point, as it is for the other methods, but is resumed before the other methods do.

\section{CONCLUSION}

In this paper three approaches were described to predict critically low voltages during a transient voltage sag caused by large rotor swings of certain generators. Voltages below a critical level can lead to further events in the system such as unintentional load tripping, which again can cause deterioration of the system condition. An early and accurate prediction of critical voltage sags is a basic requirement to execute corrective control actions. The three approaches have been assessed with respect to their abilities to correctly and early predict the crossing of a critical voltage level and the minimum of the corresponding voltage.

The results show that each of the methods can predict the voltage sags to a certain extent. While the method utilizing the phase angle of the e.m.f.'s and the method applying E-SIME allow an early detection of critical buses and low voltages, the method using voltage measurements directly allows a more accurate prediction of the voltage minimum. All in all the prediction method employing E-SIME seems to be promising. It allows early and consistent identification of critical buses and the prediction of the voltage sag minimum is sufficiently accurate. Furthermore, the approach poses an acceptable computing time and is suitable for an online application. Together with indicators providing insight on type, size and location for control actions, the method could be part of a closed-loop emergency control. For such an application the time delay, due to gathering of measurements, becomes crucial.

\section{REFERENCES}

[1] M. H. Bollen, Understanding power quality problems, vol. 3 ed. New York: IEEE press, 2000.

[2] A. A. Fouad and R. Sreedhara, "Transient Voltage Dip Analysis using the Transient Energy Function Method," in Proceedings of the Twenty-Second Annual North American Power Symposium. IEEE Comput. Soc. Press, 1990, pp. 264-273.

[3] F. Dominguez, A. S. Debs, and J. Anasis, "Transient voltage dip in power systems: computation and sensitivity analysis using the hybrid method," in Decision and Control, 1992., Proceedings of the 31 st IEEE Conference on, 1992, pp. 576-581 vol.1.

[4] Y. Xue, T. Xu, B. Liu, and Y. Li, "Quantitative assessments for transient voltage security," Proceedings of the 21st International Conference on Power Industry Computer Applications. Connecting Utilities. PICA 99. To the Millennium and Beyond, pp. 101-106, 1999.

[5] A. Tiwari and V. Ajjarapu, "Contingency assessment for voltage dip and short term voltage stability analysis," 2007 iREP Symposium - Bulk Power System Dynamics and Control, pp. 1-8, Aug. 2007.

[6] D. Shoup, J. Paserba, and C. Taylor, "A survey of current practices for transient voltage dip/sag criteria related to power system stability," IEEE PES Power Systems Conference and Exposition, 2004.

[7] P. Kundur, Power System Stability and Control, N. J. Balu and M. G. Lauby, Eds. McGraw-Hill Inc., 1994.

[8] M. I. Jordan, "Notes on recursive least squares," 1998

[9] B. Lindoff, "On the Optimal Choice of the Forgetting Factor in the Recursive Least Squares Estimator," 1997.

[10] M. Pavella, D. Ernst, D. Ruizvega, and D. Ruiz-Vega, Transient Stability of Power Systems: A Unified Approach to Assessment and Control. Kluwer Academic Publishers, 2000.

[11] M. Glavic, D. Ernst, D. Ruiz-Vega, L. Wehenkel, and M. Pavella, "E-SIME - A Method for Transient Stability Closed-Loop Emergency Control : Achievements and Prospects Fundamentals of E-SIME," in 2007 iREP Symposium - Bulk Power System Dynamics and Control, 2007.

[12] G. Rogers, Power System Oscillations. Springer, 2000. 